\title{
Der Erfolgscode
}

\author{
Fredmund Malik
}

Management ist nicht in erster Linie Management von anderen Personen, wie es immer noch verstanden wird: Management ist vor allem Management der eigenen Person. Wer sich selbst nicht zu führen vermag, hat keine Chance, andere $z u$ führen.

Management ist die wichtigste Funktion in einer modernen Gesellschaft. Aber nicht nur das Management in der Wirtschaft, also von Unternehmen, ist wichtig, sondern das gilt für alle Arten von Institutionen der Gesellschaft. Auch die Institutionen des Gesundheitswesens, des Bildungssystems, der Kultur, Verwaltung und Politik benötigen richtiges und gutes Management.

Darüber hinaus ist eine neue Entwicklung zu verzeichnen, die Management auch für die einzelne Person zu einem Schlüssel für Leistungsfähigkeit und Erfolg und über diesen Weg vielleicht sogar für persönliches Glück macht. Was die allgemeine Schulpflicht vom 17. Jahrhundert an bedeutete, muss für das 21. Jahrhundert ergänzt und erweitert werden durch Managementkenntnisse.

Die Institutionen, die bis vor kurzem lebensbestimmend und lebensgestaltend waren, haben innerhalb kür-

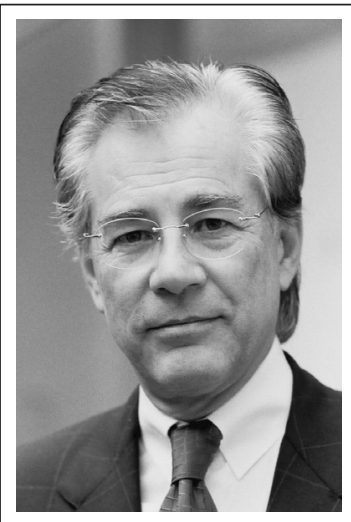

Prof. Dr. Fredmund Malik ist Leiter des seit über 30 Jahren bestehenden Malik Management Zentrum St. Gallen. Malik zählt zu den meistgelesenen europäischen Management-Autoren. In zahlreichen Büchern - darunter der Bestseller »Führen - Leisten - Leben " - und in seinem seit über zehn Jahren erscheinenden "Malik on

Management-Letter « gibt er Einblicke in das, was Führungskräfte brauchen.

Internet http://www.malik-mzsg.ch

willkommene Entschuldigung für viel tiefer liegende, grundsätzliche Schwierigkeiten. Die Bedeutung der Kirchen für die Lebensgestaltung und Lebensführung der Menschen steht auf dem Prüfstand. Die Symptome für eine neue Erstarkung sind vorläufig noch schwach und auf große Anlässe, wie Papstbesuche, beschränkt. Zu ähnlichen Ergebnissen kommt man für alle Organisationen, die Lebenshilfe und Lebensorientierung geboten haben, die Familie, das Dorf, der Verein.

Zum ersten Mal in der Geschichte der Menschheit ist der einzelne Mensch weitgehend auf sich allein gestellt. Vielleicht wird deshalb so viel von »Networking « gesprochen. Jeder muss sich selbst tauglich für die Gesellschaft machen, er oder sie muss sich selbst dort sozial brauchbar machen, wo die Schulen und andere Systeme ihre Dienste nicht mehr zu leisten vermögen. Die Menschen, besonders die jungen, müssen ihr Leben selbst managen. Das passiert nicht von allein; es muss erlernt werden.

Management ist nicht in erster Linie Management von anderen Personen, wie es immer noch weithin gesehen und in Ausbildungsveranstaltungen vermittelt wird. Management ist vor allem Management der eigenen Person. Wer sich selbst nicht zu führen vermag, hat von vornherein keine Chance, andere zu führen.

Management heißt wirksam werden, es ist der Beruf der Wirksamkeit und des Erzielens von Ergebnissen. Es ist die
Management sich als untauglich erweisen, Probleme zu lösen. Der Mangel an Geld ist nur Begleiterscheinung und 
Transformation von Ressourcen in Resultate und Nutzen, und die wichtigste Ressource ist heute Wissen.

Wissen für sich reicht aber nicht, davon gibt es überall genug. Der eigentliche Schlüssel ist die Nutzung von Wissen.

Ausbildung und Bildung genügen nicht. Für Klugheit wird niemand mehr bezahlt, nur für das, was er und sie mit der Klugheit bewirken, für die Ergebnisse. Talente, Erfahrung und Fähigkeiten, auch die vielgeforderten Sozialkompetenzen sind bedeutungslos, solange sie nicht genutzt und in Resultate umgesetzt werden. Das wird im Schulsystem aber bis auf die höchsten Stufen nicht vermittelt. Je anspruchsvoller ein Studium ist, umso weniger bleibt Zeit, jene Praktiken zu vermitteln, die einen im Beruf erfolgreich machen.

Wer mehr von Management versteht als andere, hat zwar keine Garantie für Karriere und Erfolg, aber die besten Voraussetzungen dafür. Umgekehrt ist ein Mangel an Managementkenntnissen fast eine Garantie für Misserfolg. Management als Transformation von Bildung in Ergebnisse ist der Schlüssel zum persönlichen Erfolg. Es ist in Analogie zum genetischen Code der Lebensfähigkeit der soziale Code für Lebenstüchtigkeit.

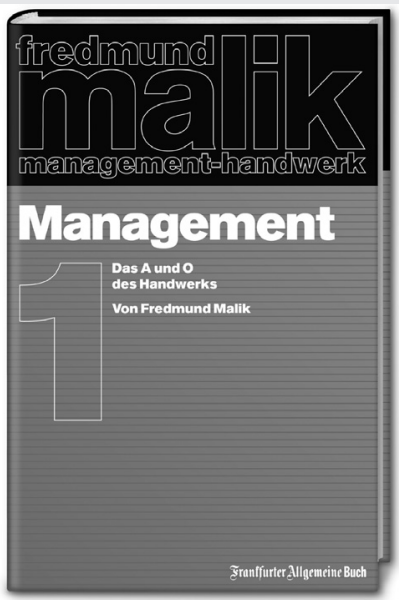

Fredmund Malik: Management. Das A und $O$ des Handwerks. Verlag FAZ-Institut, Frankfurt am Main 2005. 280 Seiten. 39,90 Euro. ISBN 3-89981-071-6.

Management ist ein Handwerk, das klare Regeln hat und lernbar ist, wie jede andere Profession auch. Die Betriebswirtschaftslehre und ei-

nen MBA-Abschluss braucht man dazu nicht. Das postuliert Fredmund Malik im ersten Band seines auf sechs Bände konzipierten Management-Lehrbuches. Richtiges Management ist universell, invariant und unabhängig von Kultur. Alle wirklich wirksamen Institutionen funktionieren auf dieselbe Weise; ob Philharmonisches Orchester oder Wirtschaftsunternehmen: Gutes Management ist überall gleich. Der Manager hat dabei mit der Betriebswirtschaftslehre ebenso viel zu tun wie der Arzt mit dem Bluttest: Es sind lediglich Mittel zum Zweck. Der für seine provokanten Thesen bekannte Managementvordenker konzipiert in dieser praxisorientierten Gesamtdarstellung ein Standardmodell für richtiges, funktionierendes Management. Es folgen die Bände: Corporate Governance, Strategie, Struktur, Kultur und Führungskräfte.

\section{Aktuelle}

Neuerscheinung

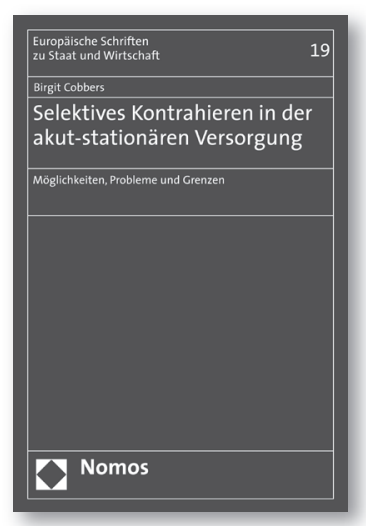

Selektives Kontrahieren in der akut-stationären Versorgung

Möglichkeiten, Probleme und Grenzen

Von Dipl.-Vw. Birgit Cobbers, Bundesministerium für Gesundheit 2006, 198 S., brosch., 39,- $€$, ISBN 3-8329-2096-X (Europäische Schriften zu Staat und Wirtschaft, Bd. 19)

Die Beziehungen zwischen Krankenhäusern und gesetzlichen Krankenversicherungen in Deutschland sind derzeit noch weitgehend von einem beidseitigen Kontrahierungszwang und einem Kollektivvertragssystem auf Kassenseite geprägt. Die Autorin untersucht zunächst die theoretischen Vor- und Nachteile eines Einzelvertragssystems.

In überversorgten Krankenhausmärkten können die Krankenkassen Preisreduktionen und/oder Qualitätsverbesserungen von den Krankenhäusern verlangen. Ob die Krankenkassen bei ihrer Auswahl von Krankenhäusern auch die Präferenzen der Versicherten berücksichtigen, hängt davon $a b$, wie intensiv der Wettbewerb auf dem Krankenversicherungsmarkt ist. Empirische Erfahrungen in den USA bestätigen die theoretischen Prognosen weitgehend.

Selektives Kontrahieren in besonders wettbewerblichen Märkten trug zu einer deutlichen Kostendämpfung bei, die Evidenz zur Entwicklung der Qualität ist ambivalent. Überlegungen zur praktischen Ausgestaltung von Vertragswettbewerb in der akut-stationären Versorgung in Deutschland vervollständigen die Untersuchung.

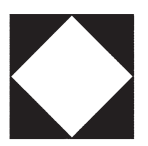

\section{Nomos}

Bitte bestellen Sie bei Ihrer Buchhandlung oder bei Nomos Verlagsgesellschaft

76520 Baden-Baden | www.nomos.de 